\title{
Diagnostic Dilemmas and Management Challenges of Organic Brain Syndrome with Psychiatric Semiology
}

\author{
Mahmoud A. Awara ${ }^{1}$, Hamdy F. Moselhy ${ }^{2}$, Maryam S. Sirry ${ }^{3}$, Manal O. Elnenaei ${ }^{4}$ \\ ${ }^{1}$ Department of Psychiatry, Dalhousie University, Halifax, Nova Scotia Health Authority, Canada; ${ }^{2}$ Department of Psychiatry, \\ Erada Centre for Treatment and Rehabilitation, Dubai, United Arab Emirates; ${ }^{3}$ Faculty of Medicine, Cairo, Egypt; ${ }^{4}$ Department of \\ Pathology, Dalhousie University, Halifax, Nova Scotia, Canada.
}

\section{Corresponding Author:}

Dr. Mahmoud A. Awara

Email:mahmoud.awara@nshealth.ca

This is an Open Access article distributed under the terms of the Creative Commons Attribution License (creativecommons.org licenses/by/3.0).

Received : February 8, 2017

Accepted : April 8, 2017

Published : April 30, 2017

\begin{abstract}
Background: Organic brain syndrome could resemble in a protean manner the psychopathology of different psychiatric disorders. Case Report: To share with the scientific community the challenges that professionals face when managing a complex case and also to shed some light on the interface between organic brain syndrome and it's psychiatric and somatic sequelae as illustrated in this case report of a forty-year-old male patient. This case is of particular interest due to the inexplicable multiple somatic and psychiatric complaints that led to numerous hospital admissions and countless investigations. Conclusion: It presented significant clinical challenges to clinicians who have been involved in the management of this patient over the past thirty years. The quandary was in diagnosis and subsequently in treating a difficult to conclude about condition that can be traced back to this patient's early childhood.
\end{abstract}

Keywords: Brain, Neurocognitive Disorders, Psychopathology, Psychotic Disorders.

\section{Introduction}

Despite the dichotomy between neurological and psychiatric disorders, their presentation may sometimes be difficult to demarcate. Psychiatrists deal with disorders of the mind and neurologists deal with disorders of the brain, although they are indivisible and often interconnected in real clinical life. Therefore, it is not possible to make absolute distinction between mental/psychiatric symptoms that arise from organic brain disease and those arise from functional brain disorders. This case-report is an example of how the gap between neurology and psychiatry may be bridged.

\section{Case Report}

Mr. $\mathrm{S}$ is a 40-year-old psychiatric patient who presented to the Emergency Department (ED) following deterioration of his mental state.
Apparently, he had stopped responding to phone calls from his parents and at their request his exboyfriend made welfare check and found Mr. S to be confused, talking to himself in an incoherent manner and his apartment was uncharacteristically in a state of complete disarray. Following assessment of his mental state in the ED, Mr. $\mathrm{S}$ was admitted to a medical ward to investigate the cause of his confusion. Blood tests showed slightly elevated creatinine and sodium levels which normalized prior to his transfer to the acute psychiatry ward. During his stay on the medical ward, Neuroleptic Malignant Syndrome (NMS) was suspected as an initial diagnosis. This was then ruled out and a diagnosis of delirium of unknown origin was eventually given. Within two months of his admission to an acute psychiatric ward he was transferred to the psychiatric rehabilitation unit where our team became first acquainted with him. 
According to his parents, Mr. S who is a product of a full-term pregnancy and normal vaginal delivery had health problems that started early on at the age of 6 months. A noticeable increase in his head circumference at that time, led to an initial suspicion of hydrocephalus. A brain scan, however, revealed a frontal lobe hematoma, of unknown etiology. Surgical excision of this hematoma was subsequently performed, with no anticipated longterm sequelae. Mr. S experienced some difficulties with his developmental milestones mainly in the form of delayed walking (first steps at two years of age) and delayed talking (first word at the age of 18 months). He also struggled in some of his motor skills e.g. climbing stairs, but was toilet-trained at a normal age. He also struggled academically and finished high school when he was 20 years old.

Mr. S has no history of mental illness in his bloodline; he is the eldest of four siblings, all of whom had successful lives. Chronic mental health issues since the age of 12 were culminating in numerous hospitalizations and made his relationship with family members tenuous; though his parents maintained their support and visits. Mr. $\mathrm{S}$ has worked in a range of jobs in the past, including grocery store cashier, cleaner, dietary aid and data entry clerk. His employments were interrupted by the multiple hospitalizations, and his last job had been five years prior to his recent admission to a psychiatric ward. When he was in his mid-twenties, he had a heterosexual relationship that lasted for one year before he changed his sexual preference. At the age of 34 years he lived with a boyfriend with whom the relationship lasted for four years.

His first contact with psychiatric services was at the age of 12 years and his first admission at the age of 14 years, which was triggered by an overdose of tricyclic antidepressant medication. His parents narrated that this was a serious and unpredictable overdose that occurred on his mother's birthday. Further to this attempt, Mr. S made threats of self-harm but never acted on them.
Mr. S is not known to have illicit drug or alcohol misuse problems. Mr. S' parents reported that his inexplicable range of behaviors began at an early age and that he was in general an anxious child. One example of his strange behavior was when he called the fire brigade at the age of seven to report a fire that did not take place. He also made unfounded allegations of being assaulted by a girl at school at the age of twelve. Moreover, in his late teens, he called the police on a number of occasions claiming domestic violence perpetrated by his father, who was at home at the time of these accusations. Indeed, similar recurring behavior was noted in more recent years when he would call the police during escorted visits from the hospital to his apartment, claiming that he had been kidnapped. He also made further claims that he had been assaulted and detained in hospital against his wish, despite that the relevant admissions were informal and that he was free to self-discharge; when quizzed, Mr. S would deny having contacted the police.

Mr. S has had, in total, about 30 psychiatric admissions to child and adolescents service as well as general adult inpatient, both acute and rehabilitation services. On occasions, he would attempt to get himself readmitted, on the same day of his discharge, into the psychiatric facility in another province. He incurred $\$ 12,000$ in debts over transportations between provinces. Due to Mr. S's numerous admissions and multiple presentations, he attracted multiple diagnoses that included bipolar affective disorder (BAD), schizoaffective disorder, neurocognitive disorder, autistic spectrum disorder, adjustment disorder, obsessional compulsive disorder, delirium NYD (Not Yet Determined), and cluster B \& C personality traits.

On further examination of the nature of $\mathrm{Mr}$. $\mathrm{S}$ ' symptoms, he appeared to have unpredictable shifts in his mood, during which he would behave in an automatic robotic manner. Examples of 
such behavior includes removing all the kitchen utensils then returning them to the drawers in the rehabilitation unit, invading others' personal space, staring at things in a trance-like state as well as speaking to himself forcefully and loudly. On direct inquiry, Mr. S indicated that he does not know why he talks to himself but he denied that he has ever heard or responded to voices, he also denied experiencing paranoid psychosis. Symptoms suggestive of a diagnosis of BAD were also denied. However, he held on to the belief that he was suffering from BAD according to a diagnosis he received at the age of 14 years. Of note, he had been on lithium for many years, though his parents reported that it did not reduce the frequency of his hospitalizations or the level of support he required in the community, since he had always been supported there either by his parents or by the individuals with whom he had a relationship.

Mr. S was later maintained on quetiapine (700 mg XR) and sodium valproate (500 mg TID) during his stay on the rehabilitation inpatient unit. The latter was prescribed after he had two consecutive falls witnessed by the staff, during which he injured his forehead and required a number of stitches. Mr. S continued to act in an irrational and inexplicable manner e.g. calling the police to claim he was kidnapped in his apartment whilst he was an inpatient on the unit, or reporting being detained when his status was voluntary, and would trigger off all the fire alarms on the unit. Furthermore, he would set up a target for himself e.g. register for a course at the college and set up a goal that he could not commit himself to, due to the previously described challenges; he would subsequently act out in an abusive manner towards the treatment team.

Mr. S had serial cognitive assessments from theage of 16years; heundertookneuropsychological assessments in 1992 and in 1999. His overall intellectual functioning was found to be in the "lower limits of the low normal range" in 1992 and in the "high end of the borderline range" in 1999. His cognitive profile was "suggestive of moderate chronic cerebral dysfunction of a diffuse nature with the anterior regions somewhat are more involved than the posterior regions, and the left cerebral hemisphere more affected than is the right". Another neuropsychological testing was conducted in 2015, in which Mr. S's overall level of intellectual functioning was determined to be in the extremely low range and fell in the 1st percentile $(\mathrm{FSIQ}=65)$. This means that he performs equal to or better than only $1 \%$ of same-age peers on intellectual testing. He showed widespread deficits (borderline to impaired scores) in the majority of areas assessed, including attention, psychomotor processing speed, and executive functions (e.g. working memory, problem solving, planning, reasoning, and verbal fluency). Memory for verbal information was also impaired, however, Mr. S showed relative strength (low average) on one of two tasks involving memory for visual material, in keeping with his low average performance on certain measures of visual spatial processing. Language and academic skills were mixed, with average to low average knowledge of vocabulary, spelling, and basic arithmetic but deficits in reading comprehension and math reasoning (borderlineimpaired). It appeared that overall IQ might have possibly declined since 1992, as it was described at that time as being in the "lower limits of the low normal range".

In 2015, an occupational therapy assessment was undertaken using the (AMPS) Assessment of Motor and Process Skills [1]. This is an observational assessment enabling simultaneous evaluation of motor and process skills and their effect on a person's ability to perform domestic or personal daily living skills required for community living. The results were analyzed to provide profiles of performance and measures of motor and process skills. Mr. S's performance on AMPS indicated 
that his functional capacities were within the level for independent living with the need for assistance.

The bedside assessment of prefrontalexecutive function [2] was employed, which is a screening tool that was presented at the 2015 American Psychiatric Association'Annual Meeting in Toronto, Ontario. Its purpose was to provide assessment tasks that allow the examiner to identify potential difficulty in areas of executive functioning skills with the intention of deducing why a client may have difficulty completing certain tasks. Results of this screening indicated that Mr. S has had various cognitive challenges that could have a significant impact on his functional abilities. The test revealed challenges with memory, cognitive flexibility, motor and spatial planning, attention and reasoning.

Due to the nature of his multiple presentations and history of brain surgery, Mr. S was referred to a neurologist and to an epileptologist. Main findings were that eye movements pursuit was saccadic and mildly hypometric. Fundoscopy revealed normal discs with venous pulsations. Motor examination revealed normal bulk and strength in all muscle groups of the extremities, whilst tone showed mild wrist cog-wheeling only present with reinforcement. Sensory examination for pinprick, vibration and proprioception was unremarkable as was cerebellar and gait examination which included toe, heel and tandem gait. Romberg was negative. Deep tendon reflexes were $2+$ and symmetric with down-going toes bilaterally. Neurological consultations concluded that Mr. S' presentations were not that of any type of epilepsy syndrome.

Fig.1 shows the fluctuation in plasma sodium and urea levels between 2010 and 2015, during which time the patient was on lithium therapy. There appears to be a period of time in 2010 where he was in a state of hemo-dilution as evidenced by low-to-low normal urea and sodium

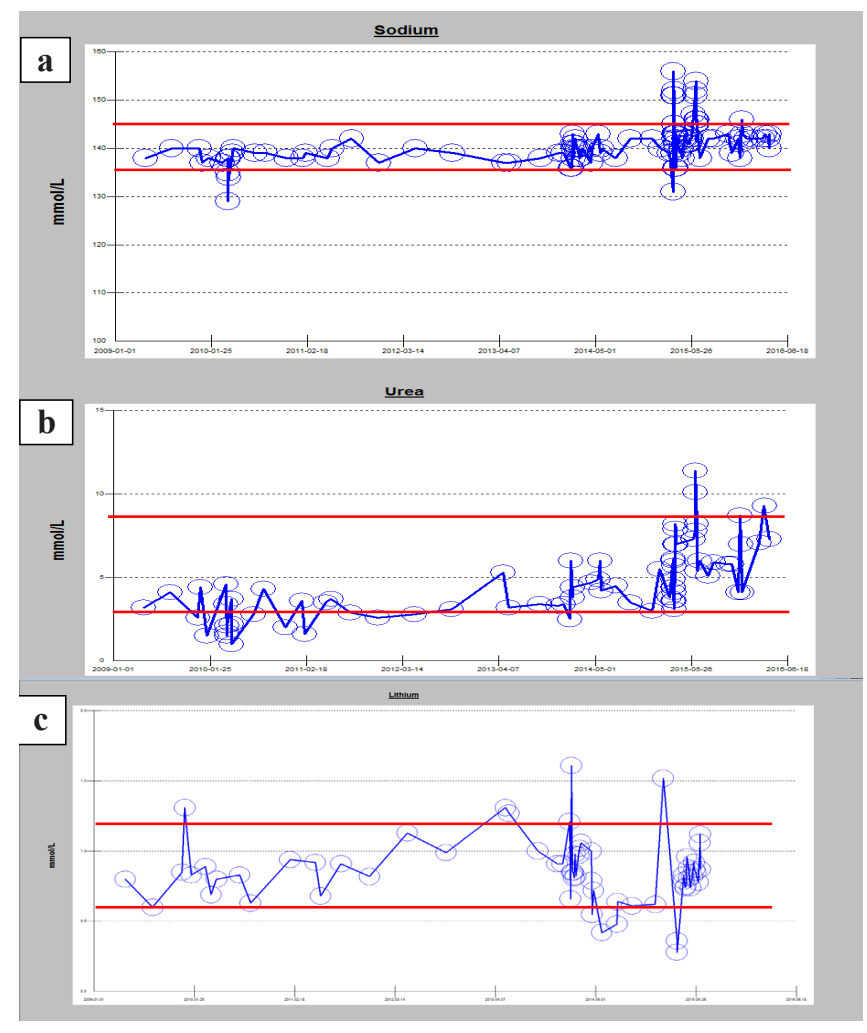

Fig.1(a): Plasma sodium levels between May 2009 - April 2016, (b): Plasma urea levels between January 2009 to April 2016, (c): Serum lithium levels from May 2009 to June 2015 after which lithium therapy had been discontinued. The two solid lines within each graph represent the reference interval for each test (therapeutic range for lithium).

levels. Throughout 2015 however he appeared to be in a state of hemo-concentration most of the time but which was intermingled with shorter periods of hemo-dilution. From his behavior observed during these intervals it was concluded that the fluctuations in these levels were as a result of him drinking fluids excessively during the peak of his lithium therapy, which could have triggered the undue thirst. As the lithium doses were tapered down over 2015, this trigger appears to have been lost resulting in a rebound temporary state of selfinduced fluid restriction, which did eventually resolve. There were, however, queries raised in 2015 by the different medical teams involved in his care as to whether his bizarre psychiatric/ neurologic presentations could be attributed, at least in part, to the electrolyte imbalance. 
Brain MRI that was carried out in 2015 reported significant frontal parietal atrophy [Fig.2]; while a brain CT scan that had been performed in 2014 had shown generalized atrophy that remained unchanged from a previous brain scan in 2007. Electroencephalogram (EEG) in 2015 revealed very subtle mild dysrhythmia, grade 1 , which was localized to the right temporal lobe. Standard brain perfusion SPECT scan using Technetium- HMPAO [Fig.3], revealed decreased perfusion on the left involving the temporal lobe, and the adjacent portions of the posterior aspect of the frontal lobe, the parietal lobe, and extending into the occipital lobe. Perfusion to the more anterior aspects of the frontal lobe was preserved. In the right hemisphere there appeared to be mildly decreased perfusion in the occipital lobe and high parietal lobe.

From an early age, Mr. S would contact the Emergency Services, to report his complaints and would subsequently be transferred to the ED on numerous occasions for chest pain, abdominal discomfort, worsening of neck pain, nausea and vomiting. This generated a myriad of investigations including blood tests, $\mathrm{X}$ rays and scans on his chest, abdomen, and spine that mostly reported unremarkable findings.

\section{Discussion}

This case illustrates the interface between organic brain syndrome and its psychiatric sequelae. The patient has had a long and arduous journey within the realm of psychiatry since the age of twelve that was intermingled with presentations to the ED in view of multiple inexplicable somatic complaints. Indeed, his problems evolved in early childhood as evidenced by delayed developmental milestones and academic backwardness. Multiple brain scans (CT \& MRI) revealed gross pathological atrophy in his fronto-parietal regions, and SPECT brain scan revealed hypo-perfusion that was more prominent on the left side. In addition, neuropsychological and OT assessments revealed widespread deficits

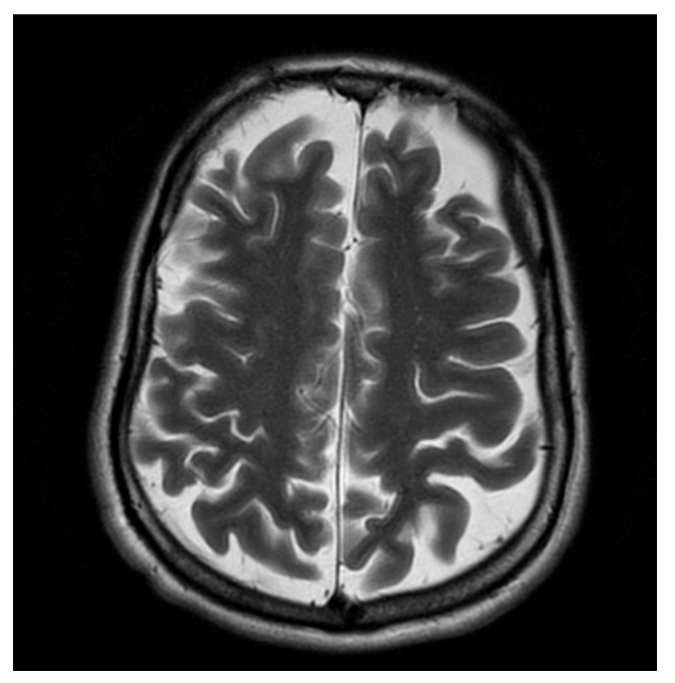

Fig.2: MRI brain scan showing frontal-parietal atrophy.

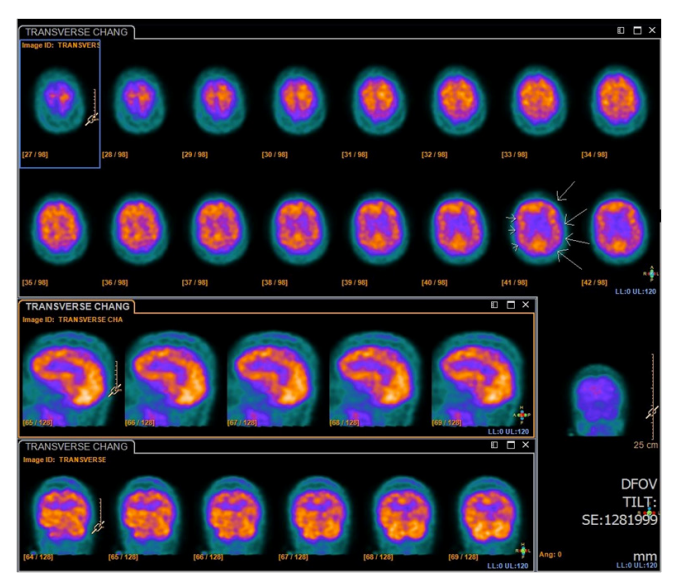

Fig.3: SPECT brain scan showing hypo-perfusion more prominent on the left side of the brain.

in brain functioning. In line with the organic nature of his psychiatric presentations, there was no history of any psychiatric disorder in his bloodline and the early onset of Mr. S's difficulties as noted by his parents was synchronous with the discovery of the hematoma in his frontal lobe.

Notably, Mr. S has had numerous presentations to the ED for inexplicable somatic complaints of chest pain and tightness, which resulted in countless investigations that yielded unremarkable findings. These somatic complaints 
appeared to happen in the context of panic attacks, associated with intense anxiety, fear and palpitations that were interpreted by the patient as a cardiac attack. A well-documented link exists between temporal lobe epilepsy (TLE) and panic disorders [3]; however, in the absence of characteristic motor activities and with negative inter-ictal EEG findings, diagnostic difficulties may arise. The increased anxiety symptoms might not be a consequence of the seizures, but rather an expression of co-morbid neurologic and psychiatric-like conditions. The mechanisms that may explain these conditions include a hyperactive hypothalamic-pituitaryadrenal (HPA) axis and its neuro-anatomic and neuro-pathologic complications, as well as disturbances in serotonergic, noradrenergic, gamma aminobutyric acid (GABA) and glutamatergic neurotransmitter systems, all of which may be interrelated [4]. This interrelationship between neurologic and psychiatric manifestations is well highlighted in a case series presented by Sazgar et al., 2003 [3]. In this patient cohort, the association of ictal panic and anxiety symptoms with partial seizures that lateralized to the right temporal lobe was demonstrated. These panic attacks semiology according to Sazgar had resulted in difficulties and delay in diagnosis of these patients.

At the age of six months, Mr. S had been diagnosed with a frontal lobe hematoma of unknown etiology that was then surgically excised but still resulted in motor, academic and executive functioning and skills deficits since early childhood. Significant frontal-parietal atrophy as demonstrated by MRI and widespread brain hypoperfusion as shown on the SPECT scan should draw attention to the nature of his organic brain pathology and the possible neurological and psychiatric consequences. Frontal network impairment may be presented with variable psychiatric symptoms, which in Mr. S's case has resulted in numerous admissions and multiple diagnoses.
Moreover, seizures of frontal lobe etiology (FLE) are often brief, frequent and occur in clusters, they start and end suddenly; auras are usually nonspecific and post-ictal confusion is not a major feature. FLE' multiple manifestations have been described and this, in addition to motor symptoms, include forced thinking, excessive vocalization, somatosensory symptoms, visceral symptoms, autonomic dysfunction, hallucinatory or illusional visual phenomena, olfactory hallucinations and pseudo-absences. Significant controversies exist regarding the localizing value of the described symptoms and conflicting reports are endless. The diagnosis is often hampered by the limited access of the scalp-EEG to the frontal extensive network with other parts of the brain and its inability to visualize discharges originating from mesial and orbitofrontal areas [5].

Frontal lobe syndrome encompasses a wide array of behavioral and cognitive disorders $[6,7]$. The presentation is of profound alteration in social conduct and personality, which predominates throughout the course of the illness; however, the form of the character change may be substantially diverse. Patients may become disinhibited, overactive and restless, with a fatuous, unconcerned affect. They may clown, pun, sing and dance, usually conforming to a restricted, stereotyped repertoire. Alternatively, patients may become apathetic and inert, lacking in drive and initiative and showing little response to stimuli. The "disinhibited" and "inert" forms represent opposite poles of a spectrum of behavioral disorder [8-10].

Due to the variability and protean nature of his presentations, Mr. S has attracted multiple diagnoses, the prevailing one was bipolar affective disorder and although he was prescribed lithium for his condition this did not reduce his symptoms or his numerous admissions. However, his affective state and behavior remained problematic. 


\section{Conclusion}

Mr. S' diagnosis and management represented a clinical conundrum to the professionals involved in his care. The evolving nature of the organic changes in his brain since early childhood and the possibility of an undetected epileptic focus in his frontal lobe could have been the mysterious culpable reasons behind his treatment resistant condition.

Contributors: MAA: manuscript writing, case management; HFM: manuscript editing, case management; MSS, MOE: critical inputs into the manuscript and literature search. MAA will act as guarantor. All authors approved the final version of the manuscript.

Funding: None; Competing interests: None stated.

\section{References}

1. Fisher AG. Assessment of motor and process skills ( $5^{\text {th }}$ eds). 2003, Fort Collins. CO: Three Star Press.

2. Benjamin S, Lauterbach M. Sheppard Pratt Health System Workshop: Bedside Assessment of PrefrontalExecutive Function. Proceedings of the APA Annual
Meeting 2015; Toronto, Ontario.

3. Sazgar M, Carlen PL, Wennberg R. Panic attack semiology in right temporal lobe epilepsy. Epileptic Disord. 2003;5:93-100.

4. Kanner, A. Depression and epilepsy: A bidirectional relation? Epilepsia. 2011;52(Suppl. 1):21-27.

5. Riggio S. Frontal lobe epilepsy: Clinical syndromes and pre-surgical evaluation. Journal of Epilepsy. 1995;8:176189.

6. Gustafson L. Frontal lobe degeneration of non-Alzheimer type II. Clinical picture and differential diagnosis. Arch Gerontol Geriatr. 1987;6:209-223.

7. Neary D, Snowden JS, Northen B, Goulding P. Dementia of frontal lobe type. J Neurol Neurosurg Psychiatry. 1988;51:353-361.

8. Jagust WJ, Reed BR, Seab JP, Kramer JH, Budinger TF. Clinical-physiologic correlates of Alzheimer's disease and frontal lobe dementia. Am J Physiol Imaging. 1989;4:89-96.

9. Orrell MW, Sahakian, BJ. Dementia of frontal lobe type. Psychol Med. 1991;21:553-556.

10. Miller BL, Cummings JL, Villanueva-Meyer J, Boone $\mathrm{K}$, Mehringer CM, Lesser IM, Mena I. Frontal lobe degeneration: clinical, neuropsychological, and SPECT characteristics. Neurology. 1991;41:1374-1382. 